\title{
Negative Symptoms and Cognitive Impairments in Schizophrenia: Two Key Symptoms Negatively Influencing Social Functioning
}

\author{
Koichi Kaneko \\ Division of Neuropsychiatry, Department of Brain and Neurosciences, School of Medicine, Tottori University Faculty of Medicine, \\ Yonago 683-8503, Japan
}

\begin{abstract}
Schizophrenia is a chronic, disabling disorder, which commonly emerges in adolescence and young adulthood. While pharmacological treatment with currently available second-generation antipsychotics exerts beneficial effects on the positive symptoms of schizophrenia, they have little effect on negative symptoms or cognitive deficits. Because these two types of symptoms are enduring, and negatively impact social functioning throughout the course of the illness, there is an urgent requirement to develop new effective therapeutic approaches to manage them. Negative symptoms have proven difficult to assess accurately because of their complexity, even with commonly used clinical rating scales such as the Scales for Assessment of Negative Symptoms (SANS). In this context, new "next-generation" assessment tools have recently been developed, which include items representing the five domains encompassed by the two established clusters of negative symptoms (diminished expression and avolition), and enable the detection of changes in severity. Despite various therapeutic approaches to alleviating negative symptoms, there are currently no established methods available for clinical practice. Cognitive deficits are also a core feature in the majority of people with schizophrenia, with impaired performance observed across many cognitive domains, including verbal memory, working memory, attention, and executive functions. Such cognitive deficits are like-
\end{abstract}

Corresponding author: Koichi Kaneko, MD, PhD

kaneko@med.tottori-u.ac.jp

Received 2018 March 26

Accepted 2018 April 23

Abbreviations: ALE, activation likelihood estimation; BACS, Brief Assessment of Cognition in Schizophrenia; BOLD, Blood oxygenation level dependent; BNSS, Brief Negative Symptom Scale; BPRS, Brief Psychiatric Rating Scale; CAINS, Clinical Assessment Interview for Negative Symptoms; CBT, cognitive behavior therapy; CI, confidence interval; CR, cognitive remediation; fMRI, functional magnetic resonance imaging; IQ, intelligence quotient; MATRICS; Measurement and Treatment Research to Improve Cognition in Schizophrenia; MCCB, MATRICS Consensus Cognitive Battery; NIRS, near-infrared spectroscopy; NMDA, N-methyl-D-aspartate; oxy-Hb, oxy-hemoglobin; PANSS, Positive and Negative Syndrome Scale; PCP, phencyclidine; PFC, prefrontal cortex; SANS, Scales for Assessment of Negative Symptoms; WCST, Wisconsin Card Sorting Test; WM, working memory ly associated with either reduced or inefficient function of related distributed neural networks. Psychosocial treatments for cognitive impairments in schizophrenia seem promising given the beneficial effects of cognitive remediation therapy on such impairments, as well as on social functioning, as substantiated in several metaanalytic studies with modest effect sizes. Furthermore, using functional neuroimaging techniques, the size of these therapy-induced beneficial changes in neurocognitive performance has been demonstrated to be correlated with the degree of the changes in brain activation during performing some cognitive tasks in the prefrontal and temporal cortices. This suggests neurobiological effects are exerted by psychosocial cognitive remediation treatments.

Key words cognitive impairment; cognitive remediation; motivation; negative symptoms; schizophrenia

Schizophrenia is a chronic, debilitating psychiatric disorder that usually manifests with frank psychotic symptoms, such as auditory hallucinations and delusions of persecution, which are referred to as positive symptoms. Negative symptoms, including low motivation and diminished verbal and/or non-verbal expression, and cognitive dysfunction may precede the emergence of these positive symptoms. However, they tend to attract less attention, and generally persist as residual symptoms following treatment.

Schizophrenia has an incidence of at least $0.7 \%$ of the population, and the age of onset generally ranges from adolescence to early adulthood, indicating that it represents a persistent burden for those with the disorder. Pharmacological treatment with currently available antipsychotic drugs is moderately to greatly effective in the relief of acute positive symptoms and for maintenance of stabilization. Nonetheless, the other two major classes of symptoms (i.e., negative and cognitive symptoms) are much less responsive to pharmacotherapy in both the first episode of psychosis ${ }^{1,2}$ and in chronic schizophrenia. ${ }^{3}$ In addition, these two problems have been demonstrated to have a detrimental impact on social functioning and quality of life in people with schizophrenia. ${ }^{4,5}$ Thus, it is important to elucidate the pathophysiology underlying 
these two classes of symptoms in schizophrenia, and to develop effective and tolerable treatment methods.

This review aims to provide a brief overview of current literature regarding negative symptoms and cognitive impairment in schizophrenia. We focus mainly on the clinical concepts and promising recovery-oriented treatment methods that specifically target these two classes of symptoms.

\section{NEGATIVE SYMPTOMS Phenomenology}

From a clinical point of view, people with schizophrenia likely exhibit both primary and secondary negative symptoms. While primary negative symptoms are part of the disease process itself, secondary negative symptoms, by definition, develop as a result of factors such as drug-induced extra-pyramidal side effects, social withdrawal due to suspicious delusions, depression, environmental deprivation, and anticipated stigma. ${ }^{6,7}$ Therefore, secondary and primary negative symptoms should be differentiated to determine the true severity of primary negative symptoms.

Although the general concept of primary negative symptoms is well accepted as a core domain of schizophrenia, its components were not adequately identified until recently despite considerable efforts. ${ }^{8}$ Studies using factor analysis have been used to develop a construct for primary negative symptoms, and consistently suggest two distinct clusters comprising five domains (Table 1). ${ }^{9}, 10$ These include: a) a cluster of diminished verbal and/or non-verbal expression (blunted affect and poverty of speech); and b) a cluster of avolition, i.e., reduced motivation and pleasure (amotivation, anhedonia, and asociality). There is a prevailing consensus for this conceptualization including five domains. ${ }^{8,9}$

Negative symptoms can be accurately separated from the other major characteristic symptoms of schizophrenia (i.e., positive symptoms, disorganized thoughts and behavior, depression, and cognitive impairment). ${ }^{9}$ This suggests that negative symptoms are likely related to different underlying pathophysiology to that underlying positive symptoms. As such, they are likely to be responsive to treatments other than pharmacotherapy with current antipsychotics. Indeed, second-generation antipsychotic medications do not adequately alleviate negative symptoms, which means early high hopes for these drugs have faded. ${ }^{1}$ To develop new effective therapeutic approaches specifically targeting negative symptoms, an evidence-based rating instrument should be developed that can measure both the severity of each construct dimension and their change over time. ${ }^{11}$
Table 1. Two main clusters and five domains of negative symptoms

\begin{tabular}{|c|c|}
\hline Cluster and domain & Domain characteristics \\
\hline A. Diminished expression & $\begin{array}{l}\text { Reduced behaviors related to the } \\
\text { expression of emotion }\end{array}$ \\
\hline 1. Blunted affect & $\begin{array}{l}\text { Reduced intensity and range of emo- } \\
\text { tional expression shown in verbal or } \\
\text { non-verbal modes of communication }\end{array}$ \\
\hline 2. Alogia & $\begin{array}{l}\text { Reduced quantity of speech and loss } \\
\text { of conversation fluency }\end{array}$ \\
\hline B. Avolition & $\begin{array}{l}\text { Loss of behaviors, interests, motiva- } \\
\text { tion, and desires }\end{array}$ \\
\hline 1. Amotivation & $\begin{array}{l}\text { Deficits in the initiation and mainte- } \\
\text { nance of goal-oriented behaviors }\end{array}$ \\
\hline 2. Anhedonia & Impaired anticipation of reward \\
\hline 3. Asociality & $\begin{array}{l}\text { Diminished interest in social interac- } \\
\text { tions with others }\end{array}$ \\
\hline
\end{tabular}

\section{Assessment}

It is important to bear in mind that most rating scales for negative symptoms necessarily require, at least in part, inferences from the patient's self-report of avolition, which requires rating subjective feelings. These inferences are probably limited by communication abilities, and an individual may have inherent difficulties in rating their own negative symptoms.

The Scale for Assessment of Negative symptoms (SANS: 25 items, covering the five subdomains of affective flattening, alogia, avolition/apathy, anhedonia/ asociality, and attention), ${ }^{12}$ and the Positive and Negative Syndrome Scale (PANSS) negative subscale items (7 items, covering blunted affect, emotional withdrawal, poor rapport, passive social withdrawal, difficulty in abstract thinking, lack of spontaneity and flow of conversation, and stereotyped thinking $)^{13}$ are commonly used to assess negative symptoms in schizophrenia. Although these two scales have been shown to be reliable and valid rating tools for measuring negative symptoms, some fundamental problems arise from the fact that they do not necessarily include the whole range of true negative symptoms, or that they may incorporate symptoms that are not currently considered as true negative symptoms. For example, the PANSS negative subscales fail to fully cover the whole range of negative symptoms..$^{10}$ Moreover, the SANS and PANSS both includes subscales for attentional deficits, which are more likely to belong to disorganization syndrome than negative symptoms. ${ }^{10}$ In addition, these scales are less sensitive to rapid changes in the severity of negative symptoms over longer observation periods of more than a month. ${ }^{10}$

To minimize these weaknesses, and to facilitate 
development of effective treatment approaches for negative symptoms in schizophrenia, the National Institute of Mental Health's Consensus Development Conference on Negative Symptoms formed a workgroup to develop new instruments that should include the five agreed-upon subdomains and enable us to detect changes in their severity ${ }^{14}$ As a result, two "next-generation" rating scales have recently been developed for use in treatment trials and research on negative symptoms, with strong convergent and discriminant validity, test-retest reliability, and internal consistency. These are: (1) the Clinical Assessment Interview for Negative Symptoms (CAINS); and (2) the Brief Negative Symptom Scale (BNSS).

The 13-item CAINS has a two-factor structure, expression (four items) and motivation/pleasure (nine items), and was intended to cover each subdomain in extensive detail. Its ratings combine measurements based on individuals' reports of subjectively experienced pleasure in relevant activities in the past week with those of anticipatory pleasure. ${ }^{15}$ Given the evidence that anhedonia in schizophrenia is probably anticipatory in nature rather than experiential, ${ }^{16}$ measuring both types of anhedonia may contribute to further elucidating such characteristics of this disorder.

The BNSS also has 13-item structure, and similar properties to the CAINS in that it assesses two types of anhedonia. However, the BNSS was intended to be concise enough to be feasible for large, multicenter trials and clinical use. ${ }^{14}$ The 13 items are organized into six subscales, and rating usually takes about 20 minutes. The most remarkable feature of its construct is that, unlike other scales for negative symptoms, it includes a "Distress" subscale, which measures the level of the absence of dysphoria, confirmed by a negative correlation between the BNSS "Distress" item and a sum of the PANSS "Depression", "Guilt", "Anxiety", and "Hostility" items. $^{14}$

Both the CAINS and BNSS are becoming more widely used, with confirmation of their good psychometric properties. A recent study comparing the two measures reported moderate to high correspondences for blunted affect, alogia, asociality, and avolition subdomains, but low correspondence for the anhedonia subdomain. ${ }^{17}$ Such a difference may arise from differences in precisely what the scales assess. For example, the CAINS items only assess the frequency of pleasure, whereas the BNSS items assess both intensity and frequency of pleasure. Differences may also stem from the characteristics of anhedonia in people with schizophrenia such that they exhibit a dissociation between the capacity for pleasure ("liking") and the frequency of seeking out rewarding activities ("wanting"). This may result in a low correlation between BNSS ratings of the intensity of past enjoyable events and CAINS ratings of the frequency of past pleasure. Given the reasons for the development of these two next-generation rating scales, they should be used to assess the efficacy of new treatment approaches for negative symptoms.

\section{Treatment}

Recovery for people with schizophrenia should include a substantial improvement in negative symptoms and cognitive deficits, because these symptoms are found to be highly stable over time and have a long-lasting serious impact on functional outcomes. ${ }^{18}$ Although there has been extensive investigations of the efficacy of various therapeutic approaches to improve functioning of people with schizophrenia by alleviating negative symptoms, there are currently no well-established therapeutic methods. ${ }^{19-21}$

Table 2 presents efficacy as pooled standardized mean differences (SMDs) for several treatments for negative symptoms, which were reported in a meta-analysis of 168 placebo-controlled trials. ${ }^{21}$ The authors of this metaanalysis argued that such an apparent positive effect does not necessarily represent a clinically meaningful effect, because it may partly be based on improvements in secondary negative symptoms or there may be a lack of beneficial effects on functioning. ${ }^{21}$

Table 2. Efficacy of placebo-controlled treatments for negative symptoms (modified from Fusar-Poli et al., 2015)

\begin{tabular}{llccrr}
\hline Treatment & $N$ & SMD & $95 \%$ CI & Z & $P$ \\
\hline Comb & 33 & -0.518 & -0.757 to -0.279 & 4.24 & $<.001$ \\
AD & 26 & -0.349 & -0.551 to -0.146 & 3.37 & .001 \\
FGA & 10 & -0.531 & -1.104 to 0.041 & 1.82 & .069 \\
SGA & 38 & -0.579 & -0.755 to -0.404 & 6.47 & $<.001$ \\
Psych & 27 & -0.518 & -0.757 to -0.279 & 4.64 & $<.001$ \\
Glut & 26 & -0.289 & -0.478 to -0.1 & 2.99 & .003 \\
\hline
\end{tabular}

AD, antidepressant; CI, confidence interval; Comb, combined treatment; FGA, first-generation antipsychotic; Glut, glutamatergic agents; Psych, psychological therapy; SGA, second-generation antipsychotic; SMD, standardized mean difference; Z, z-test. 
As an extensive and detailed description of treatment approaches for negative symptoms is not within the scope of this review, we briefly mention some specific pharmacological agents (second-generation antipsychotics, antidepressants, glutamatergic medications, and psychostimulants) and psychological interventions (cognitive behavioral therapy, CBT) in the following sections.

\section{Pharmacological treatments}

Where certain pharmacological agents appear to exert a measurably beneficial effect on negative symptoms in a clinical trial, the results should be cautiously interpreted because there are many important methodological considerations that must be taken into account. ${ }^{10}$ These include the definition, assessment, and persistence of negative symptoms, the contribution of secondary negative symptoms, the trial duration, and so on.

\section{Second-generation antipsychotics}

Pharmacological therapy with antipsychotics is the mainstay in the treatment of schizophrenia. ${ }^{22}$ However, it is generally observed that antipsychotics exert far more significant effects on positive symptoms than they do on negative symptoms or cognitive impairment. Indeed, second generation antipsychotic medications appear not to adequately alleviate negative symptoms, leading to the fading of early, strongly-held hopes for these drugs in many countries (with the exception of amisulpiride). ${ }^{1,19}$

A recent meta-analysis intended to clarify the efficacy of available treatments on negative symptoms, reporting a medium effect size of $0.579 .{ }^{21}$ However, such beneficial effects yielded by antipsychotics may possibly be produced by improving symptoms other than primary negative symptoms, e.g., asociality due to positive symptoms, suggesting that observed negative symptoms that show improvements are secondary in nature.

\section{Antidepressants}

It is reasonable that depression contributes to negative symptoms considering the conceptual proximity of its clinical expression (e.g., both negative symptoms and depression are associated with reduced emotional expression and poverty of speech), and the underlying neural substrates. ${ }^{10,23}$ Therefore, several classes of addon antidepressants have been investigated as potential treatments for negative symptoms.

Two meta-analyses have evaluated the efficacy of antidepressants in treating negative symptoms. In one study, the authors analyzed 22 publications that assessed symptoms with standardized rating scales, including the SANS total, PANSS negative, and Brief Psychiatric
Rating Scale (BPRS). ${ }^{23}$ In the other meta-analysis, the authors exclusively examined studies that enrolled participants with pronounced negative symptoms. ${ }^{24}$ Both studies reported a beneficial, medium effect size, whereas a previously mentioned meta-analysis that included any available treatment had reported a small effect size, but concluded that such small effects were not clinically meaningful. ${ }^{21} \mathrm{~A}$ major reason for this interpretation is that observed improvements in negative symptoms may have been partly based on improvements in secondary negative symptoms. ${ }^{21}$

Therefore, although the possible efficacy of antidepressants to alleviate negative symptoms appears encouraging, the inherent difficulty in distinguishing between negative symptoms and depression should be borne in mind..$^{10}$

\section{Glutamatergic agents}

A well-established neurochemical hypothesis of the pathophysiology of schizophrenia suggests it results from N-methyl-D-aspartate (NMDA) receptor hypofunction..$^{25}$ This hypothesis is based on the wellknown finding that NMDA receptor antagonists, such as phencyclidine (PCP), can produce both positive- and negative-like symptoms. ${ }^{25}$ Moreover, such antagonists also induce neurocognitive deficits similar to those observed in schizophrenia across various subdomains of neurocognition, e.g., executive processing ${ }^{26}$ and working memory. ${ }^{27}$

Several glutamatergic agents that modulate NMDA receptor function, either acting directly or indirectly at the receptors, have been examined as possible pharmacological treatments for negative symptoms. These include agonists of the glycine site of NMDA receptors (glycine) and the partial agonist (D-cycloserine), a glycine transporter-1 inhibitor (sarcosine), an uncompetitive NMDA receptor antagonist (memantine), and an anticonvulsant (lamotrigine), which inhibits glutamate release resulting in apparently paradoxical actions. ${ }^{19}$

Results of a meta-analysis in which various kinds of NMDA receptor modulators (including D-cycloserine, glycine, and sarcosine) were examined reported evidence of a small effect size for these agents as a group. ${ }^{28}$ In addition, small to medium effect sizes were observed specifically for D-serine and sarcosine. ${ }^{28}$ Therefore, agents that modulate NMDA receptor function may offer a promising treatment for negative symptoms, although positive findings are not always consistent among studies. $^{29}$

\section{Psychostimulants}

The possible therapeutic basis of psychostimulants, such 
as amphetamine-based drugs and modafinil, in alleviating negative symptoms is that they facilitate dopaminergic transmission in the prefrontal cortex (PFC), which is assumed to be reduced in schizophrenia. ${ }^{30}$

Despite the potential risks of abuse and exacerbating psychotic symptoms, a systematic review of psychostimulant treatments for negative symptoms reported that amphetamine-based drugs have a relatively low risk of aggravating positive symptoms in patients who are clinically stable and adherent to antipsychotic medications. However, this evidence is limited because of small samples and short durations of the studies included. ${ }^{31}$ Modafinil and its enantiomer armodafinil generally show only limited clinical effects for reducing negative symptoms, which might result from their weaker inhibitory actions on the dopamine transporter than amphetamine. $^{32}$

\section{Psychosocial treatment}

Current psychiatric clinical practice should promote the recovery of people with mental illness. The modern concept of recovery stresses the importance of improving subjective well-being and quality of life of patients, as well as social functioning including independent living skills, and capacity to work or study. ${ }^{33}$ Both negative symptoms and cognitive impairments are known to deleteriously impact on these functions. ${ }^{18}$ Because current pharmacological approaches exert minimal effects on these symptoms, non-somatic, psychosocial interventions are considered especially important in the management of persistent, drug-resistant symptoms. ${ }^{34}$ It is now assumed that negative symptoms are not a consequence of fixed deficits but can at least partly be considered as dysfunctional beliefs about one's cognitive and social abilities, as well as one's aptitude for pleasure. ${ }^{20,35}$ From this new viewpoint, it is possible that psychosocial treatments will have beneficial effects on negative symptoms.

Among various kinds of psychosocial treatment approaches aimed at alleviating symptoms of schizophrenia, CBT appears by far to be the most established and frequently sought approach. Therefore, in this review, discussion of the efficacy of psychosocial treatments for negative symptoms will be confined to that of CBT.

CBT for schizophrenia was originally developed to reduce positive symptoms, with a primary focus on psychotic symptoms and general approaches adopted to weaken firm beliefs. ${ }^{36}$ This is substantially different from traditional CBT for mood and anxiety disorders. As such, very few clinical studies have addressed negative symptoms as a primary outcome. The literature on the therapeutic effects of CBT on positive symptoms appears to warrant the conclusion that such psychological therapy is more effective than usual treatment. ${ }^{37,}, 38$ Nonetheless, results from clinical trials and a meta-analysis of the efficacy of CBT in treating negative symptoms of psychosis appear to be mixed..$^{20}$

Early clinical studies mostly tended to indicate beneficial effects of CBT on negative symptoms, as reported in a meta-analysis by Wykes et al., in which they reported an effect size of $0.44 .{ }^{37}$ However, the majority of early published clinical trials examining the efficacy of CBT for schizophrenia assessed negative symptom severity as a secondary outcome. In contrast, more recent meta-analytic studies conducted to take into account various sources of bias on effect size fail to support the efficacy of CBT to improve symptomatic measures. ${ }^{20,39} \mathrm{In}$ these meta-analyses, effect sizes were calculated using Hedges' $g$, the standardized difference between means, and reported effect sizes of CBT for negative symptoms were as following: In studies with negative symptoms as a secondary outcome, Jauhar et al. ${ }^{39}$ reported an effect size of 0.13 (95\% confidence interval, CI, 0.01 to 0.25 ) and Velthorst et al. reported an effect size of 0.093 (95\% CI -0.028 to 0.214 ) for CBT for negative symptoms. Similar effect size of 0.157 (95\% CI -0.10 to 0.409 ) has been reported in studies that focus on negative symptoms as a primary outcome. ${ }^{20}$

Such large differences between older and newer studies in reported effect sizes for CBT cannot be attributed to difference in the demographic properties of participants, study samples, number of treatment sessions, characteristics of the control group, number of treatment ingredient, or illness duration. ${ }^{20}$ However, there appears to be three plausible reasons for the observed decline in effect sizes suggested by the two recent meta-analytic studies mentioned above. First, various sources of potential bias observed in older CBT trials, such as insufficient masking when assessing outcome measures, likely have profound effects on their results as demonstrated by Jauhar et al. ${ }^{39}$ Second, newer studies more often target a single clear-cut outcome, such as auditory hallucinations, which might result in smaller improvements in negative symptom measures. Third, results of older behavior-oriented studies may suggest CBT is more effective in alleviating negative symptoms than more recent cognition-oriented studies. This possibility suggests the importance of individuals' active participation in social activities.

In this context, it is especially noteworthy that a recent 18-month recovery-oriented cognitive therapy trial targeting low-functioning people with schizophrenia successfully yielded clinically significant improvements in negative symptoms and global functioning. ${ }^{40}$ The therapy was intended to be flexible regarding the plans 
and schedules based on the participant's needs and progress. It was also aimed at stimulating their interest and motivation to focus on achievable goals based on the available time.

The efficacy of this promising cognitive therapy to alleviate negative symptoms suggests that these apparently persistent symptoms may arise at least partly from dysfunctional beliefs about an individual's own abilities, and that they are not completely determined by hedonic deficits inherent to schizophrenia. ${ }^{20}$ Because dysfunctional beliefs have been shown to mediate the association between neurocognitive impairment and negative symptoms, ${ }^{40,41}$ such nihilistic beliefs should be a target of psychological treatment to aid recovery. However, more research is required to draw firm conclusions about the efficacy of this kind of cognitive therapy for persistent negative symptoms.

\section{COGNITIVE IMPAIRMENT}

The dominant view in the past was that people with schizophrenia have limited, if any, neuropsychological impairments resulting from psychotic symptoms. However, compromised neurocognition has more recently been established as a core feature of schizophrenia. ${ }^{42}$ Cognitive impairments have been observed in the majority of patients with schizophrenia, but not all. ${ }^{43}$ It is particularly important to note that, in contrast to psychotic symptoms, cognitive impairment predicts a range of poor functional outcomes, e.g., verbal memory is associated with all types of functional outcome examined. ${ }^{44}$

From a longitudinal perspective, cognitive deficits are evident before florid symptoms emerge with substantial cognitive impairments already present at the time of first psychiatric contact. ${ }^{45}, 46$ These impairments have been shown to persist after psychotic symptoms have remitted, indicating that cognitive impairments are present throughout all phases of the illness, including in clinical high-risk cohorts, ${ }^{47}$ first episode patients, ${ }^{48}$ and patients with chronic schizophrenia. ${ }^{49}$ A systematic review of 58 studies of the associations between neurocognitive impairments and psychotic psychopathology has documented that negative and disorganized dimensions are modestly associated with cognitive deficits, whereas positive and depressive dimensions are not. ${ }^{50}$ This suggests that impaired neurocognition is not secondary to positive symptoms.

\section{Profile of neurocognitive deficits Generalized cognitive deficits}

General neurocognitive ability has been measured in schizophrenia by either measuring the intelligence quotient (IQ) or composite scores obtained from neuropsychological test batteries comprising multiple tests that assess different cognitive abilities. Standardized IQ tests primarily focus on the measurement of crystallized abilities ${ }^{51}$ whereas neuropsychological test batteries mainly measure multiple cognitive abilities including verbal memory, working memory, attention, and executive functions.

With these measures, it has been shown that the illness results in a generalized impairment of cognitive performance across many cognitive domains, which are affected to a fairly similar extent. This naturally leads to the hypothesis that cognitive impairment inherent to schizophrenia appears to be global in nature. This hypothesis has been supported by the results of two large-scale meta-analytic studies. ${ }^{52,53}$ These studies have reported "severe" cognitive deficits based on a Cohen's $d$ of -0.92 and -0.98 .

\section{Specific cognitive deficits}

As general neuropsychological tests often do not assess pure domains of neurocognitive ability, the results yielded by such tests should be interpreted carefully. Despite the apparent global nature of neurocognitive impairments in schizophrenia, a group of experts who worked on the Measurement and Treatment Research to Improve Cognition in Schizophrenia (MATRICS) project proposed seven key domains of cognitive deficits in the condition, which are assumed to be most impaired and most strongly correlated with outcome. These were working memory, attention/vigilance, verbal learning and memory, visual learning and memory, reasoning and problem solving, speed of processing, and social cognition. ${ }^{54}$ In this section, brief descriptions of these cognitive domains are provided mainly with reference to Keefe and Harvey. ${ }^{55}$

\section{Vigilance and attention}

Sources of attention can be broken down into three functions: alerting, orienting, and executive control..$^{56}$ Vigilance means the ability to maintain attention over time. People with schizophrenia can have difficulties in being fully engaged in everyday conversations with others and in following important instructions at work. ${ }^{42}$ These cognitive deficits appear related to functional outcomes, such as community functioning and skills acquisition. ${ }^{42}$

\section{Verbal learning and memory}

To encode new information, store it over time, and retrieve it requires memory functions. Historically, memory was not regarded to be severely impaired in 
schizophrenia. However, modern, precise, standardized neuropsychological measurements applied in schizophrenia research have suggested severe deficits in memory ability with effect sizes of less than $-1.0 .^{42}$ People with schizophrenia generally exhibit larger impairments in encoding than in retrieval. ${ }^{42}$ Moreover, verbal memory impairments are associated with real-world functioning.

\section{Visual learning and memory}

Visual memory describes the relationship between visually perceived information and its encoding, storage, and retrieval. The degree of deficit in these abilities in schizophrenia is usually less than that of memory. ${ }^{42}$ This cognitive ability has been shown to be moderately correlated with employment status and strongly correlated with functional capacity.

\section{Reasoning and problem solving}

Some of the most commonly used and most well-known cognitive tests include the Wisconsin Card Sorting Test (WCST) and the Stroop test. People with schizophrenia exhibit poor performance on both these tests. The WCST is generally interpreted as assessing abstraction/ problem-solving skills and the ability to efficiently shift between adopted strategies. However, performance on the task does not reflect a single measure of executive functions but various kinds of cognitive abilities. People with schizophrenia often display poor performance on this task, which reflects difficulties in adapting to changes in the world around them. ${ }^{42}$

\section{Speed of processing}

Processing speed is defined as the speed at which cognitive operations can be executed and it likely affects various neurocognitive processes, suggesting that impairments in this cognitive function are non-specific. Such deficits have been found to correlate with various measures of functioning, including daily life activities, job tenure, and independent living status. ${ }^{42}$ In a recent meta-analysis of cognitive deficits, this measure was reported to be the most severe impairment in schizophrenia. ${ }^{53}$

\section{Working memory}

Working memory (WM) has been often defined as the temporal storage and manipulation of incoming information to perform complex cognitive tasks. ${ }^{57}$ Due to remarkable correlations between WM measures and other neurocognitive abilities, impaired WM has been regarded as a core component of cognitive impairments in schizophrenia, ${ }^{58}$ and appears to be related to employment status and job tenure. ${ }^{55}$ Verbal or spatial working memory tasks conducted in a large number of functional magnetic resonance imaging (fMRI) studies have suggested altered levels of activation in the prefrontal and anterior cingulate cortical regions, and posterior parietal cortex..$^{59}$

\section{Social cognition}

Social cognition is defined as the ability to construct representations of the relationships between oneself and others, and to use those representations flexibly to guide social behavior, which is qualitatively distinct from neurocognition. Research of schizophrenia has mainly focused on theory of mind (making inferences about another's intentions), social perception, emotional processing, and attribution style, with reports of substantial impairments across these subdomains. Impaired social cognition is related to social impairments and is shown to largely mediate the effects of neurocognition on functional outcome. ${ }^{60}$

\section{Assessment}

Assessment of neurocognitive function appears to be less challenging than that of negative symptoms because there already exist established test batteries for the assessment of neurocognitive abilities, for which reliability and validity have been demonstrated.

The most well-known and most comprehensive test battery is the MATRICS Consensus Cognitive Battery (MCCB), ${ }^{61}$ which was intended to be used across treatment studies targeting neurocognition. This battery measures the above mentioned seven key domains of cognitive abilities with high sensitivity.

Another test battery that is less exhaustive than the MCCB and requires minimal time for completion and so is utilized widely in clinical studies is the Brief Assessment of Cognition in Schizophrenia (BACS). ${ }^{62}$ The BACS covers six neurocognitive domains of verbal memory, working memory, motor speed, attention, executive functions, and verbal fluency. This battery also provides a composite score that is calculated by averaging all of the six standardized primary measures and that can reflect treatment-induced changes due to its high reliability. ${ }^{62}$ Compared with age- and parental education-matched healthy controls, people with schizophrenia have BACS composite scores of about 1.5 standard deviations lower.

\section{Treatment}

The current ultimate goal of treatment for schizophrenia should be to facilitate recovery by improving long-term functioning of people with the illness beyond the remission of psychotic symptoms. Improving brain function 
(especially activity of the PFC during cognitive load) can result in better functional outcomes because it has been reported to be closely associated with cognitive functioning measures even when assessed with different scales functions. ${ }^{63-65}$ However, there are no approved treatments for impaired cognition, whether pharmacological or psychological.

In this section, greater emphasis is given to newly developed cognitive remediation (CR) therapy than pharmacotherapy, which has shown minimal benefits. ${ }^{55}$

\section{Second-generation antipsychotics}

Although early clinical studies reported that secondgeneration antipsychotics yielded greater cognitive benefits than did first-generation antipsychotics, results of later studies did not support these findings. Observed effect sizes were small (0.2-0.5), and this magnitude was viewed as consistent with practice and/or placebo effects. ${ }^{55}$ In addition, small cognitive benefits of secondgeneration antipsychotics in first-episode patients appeared to be related to symptom changes, although the correlation was weak. ${ }^{66}$ Therefore, the current literature suggests that antipsychotics provide limited benefits for cognition. ${ }^{55}$

\section{Cognitive remediation $(\mathbf{C R})$}

CR therapy for schizophrenia aims to improve cognitive abilities through a wide range of learning-based interventions with the ultimate goal of generalization of cognitive skills to functional skills, as well as to other untrained neurocognitive tests, and enhanced real-world functioning. ${ }^{67}$

Accumulating data indicate beneficial effects of $\mathrm{CR}$ on neurocognition despite wide variability in the remediation approaches employed. Indeed, almost all meta-analyses have demonstrated moderate ${ }^{68,69}$ to large ${ }^{70}$ effect sizes of CR on global neurocognition. Although the efficacy of CR for social functioning has been found to be generally smaller than that for neurocognitive abilities, it has also been shown to be greater when provided with adjunctive psychiatric rehabilitation (Cohen's $d=$ $0.59)$ than when given alone $(d=0.28) .{ }^{69}$ Moreover, durability of training effects have been repeatedly shown to be six months or more after training, ${ }^{71}$ which has been confirmed by two large-scale meta-analyses. ${ }^{68,69}$

From the results of these studies, it appears that there exists no conclusively demonstrated moderator variables that affect outcomes, including methodological qualities (e.g., type of control group and masking), participant characteristics (e.g., age and severity of baseline symptoms), and program characteristics (e.g., drill and practice vs. drill and practice plus strategy coaching, hours of practice, and top-down vs. bottom-up strategies). However, a meta-analysis by Wykes et al. reported that among studies providing adjunctive psychiatric rehabilitation programs, there are more beneficial effects of a CR program employing strategy coaching than other programs only applying a drill and practice approach. ${ }^{69}$ In consequence, it is currently difficult to identify key elements of CR interventions because of considerable variation among CR programs with few studies of adequate methodological quality available to date.

Although the neural mechanisms underlying beneficial CR effects on cognition and functioning currently remain unclear, recent functional or structural neuroimaging studies have reported brain changes that may be associated with $\mathrm{CR}$ effects. One systematic review included studies investigating both CR effects on cognition and longitudinal changes in neural measures that would reflect either functional neural activity or brain structure assessed at pre-treatment and post-treatment. ${ }^{72}$ This systematic review included seven functional magnetic resonance imaging (fMRI) studies, all of which reported possible $\mathrm{CR}$ effect-related brain changes. These were either an increase or a decrease in BOLD signals or functional connectivity in brain regions of people with schizophrenia, but regions did not necessarily completely overlap across the studies. These putative "CR effect-related" brain regions include prefrontal, temporal, parietal, occipital, and limbic areas, with the PFC being the most consistently reported region..$^{72}$

One near-infrared spectroscopic study with a longitudinal design also reported putative CR-related changes in WM task-elicited brain activation in the right prefrontal and temporal cortices, the magnitude of which was correlated with improvements in neurocognitive measures of verbal memory and verbal fluency (Fig. 1). ${ }^{73}$ In addition, structural changes possibly due to $\mathrm{CR}$ have also been observed in two reviewed studies, including neuroprotective effects associated with increased amygdala volume, ${ }^{74}$ and increased white matter integrity of the genu and body of the corpus callosum, which was negatively associated with the degree of normalization of heightened task-related functional connectivity. ${ }^{75}$

A meta-analysis of nine CR studies examining changes in functional activation with fMRI or positron emission tomography investigated with an activation likelihood estimation (ALE) method investigated whether CR delivered via variable methods could cause plastic changes in common neuroanatomical regions or not. ${ }^{76}$ ALE analyses comparing brain activation at two time points (pre- and post-training) revealed increased activity in the lateral and medial PFC, parietal cortex, insula, 


\section{A}

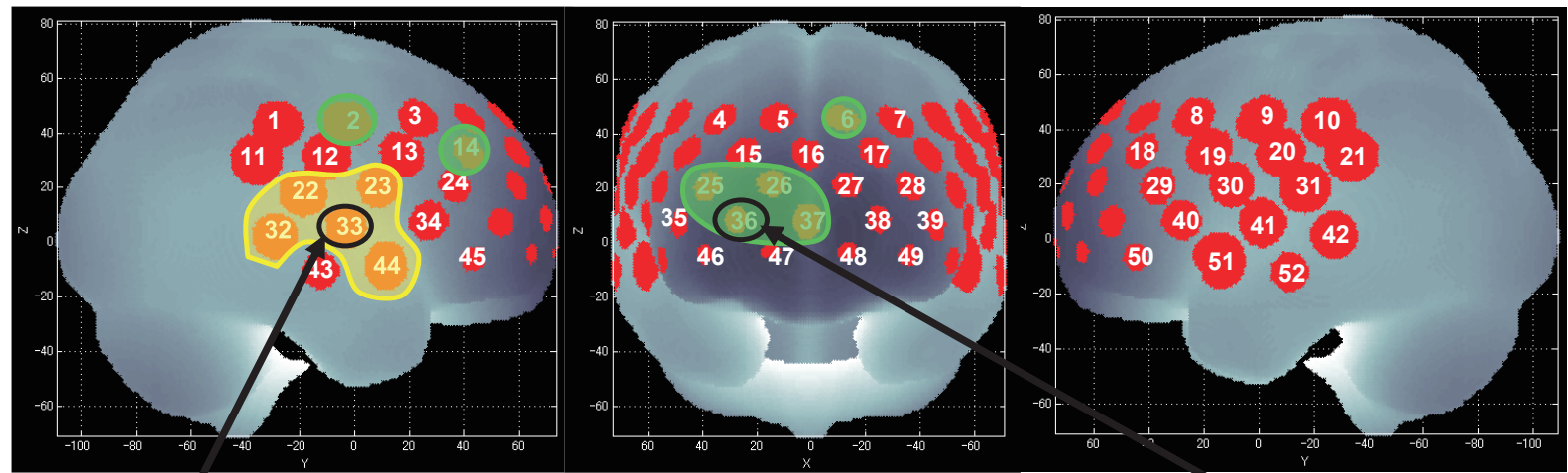

$\mathrm{B}$

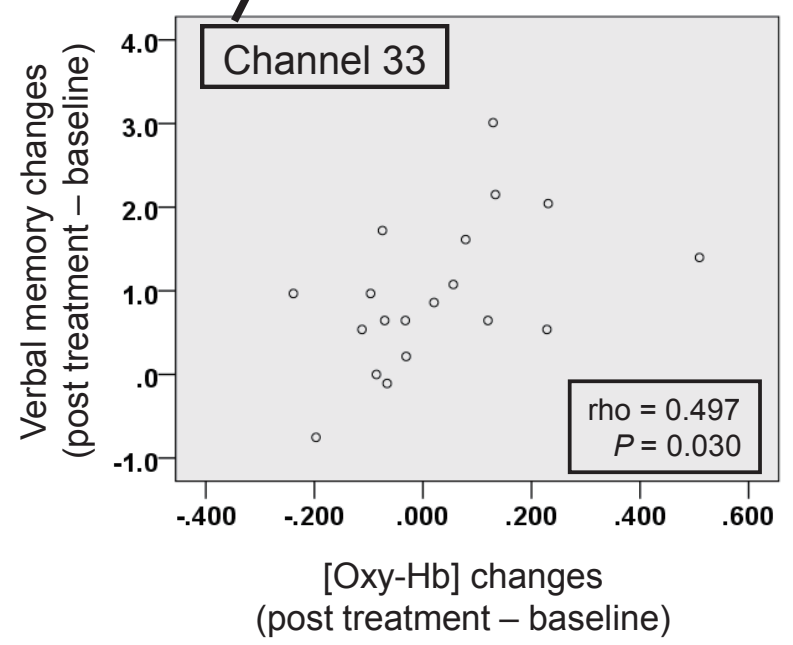

C

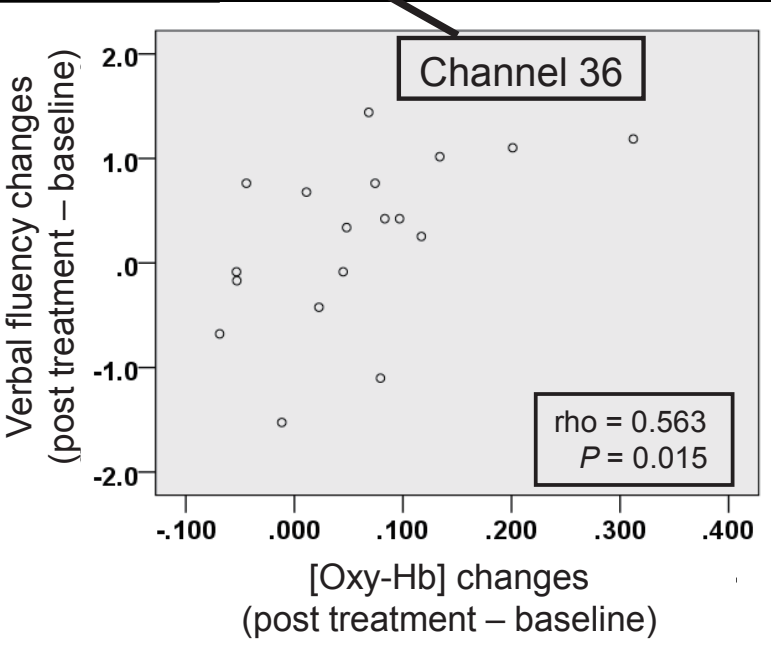

Fig. 1. The extent of improvements in performance of some neurocognitive functions following cognitive remediation (CR) are significantly correlated with the magnitude of increases in working memory task-elicited prefrontal and temporal hemodynamic responses during working memory task measured with near-infrared spectroscopy (NIRS).

(A) The relationship between cognitive function and changes in the concentration of oxy-hemoglobin ([oxy-Hb]) associated with working memory tasks before and after cognitive remediation. Red circles with the channel number in white indicate the locations of channels and, measurement points for 52-channel NIRS. Green and yellow represent the channels in which there are verbal memory and verbal fluency changes, respectively, in the Japanese version of the Brief Assessment of Cognition in Schizophrenia (BACS-J).

(B) Scatter diagrams showing the relationship between verbal memory and [oxy-Hb] changes in channel 33 (Spearman's Rho; rho = 0.497, $P=0.030$ ).

(C) Scatter diagrams showing the relationship between verbal fluency and [oxy-Hb] change in channel $36(r h o=0.563, P=0.015)$. The locations of near-infrared spectroscopy channels were probabilistically estimated and anatomically labeled in the standard brain space.

and the caudate and thalamus. It is particularly noteworthy that CR-related, activated regions in the left PFC and thalamus partially overlap with previously identified areas associated with deficits in WM, executive control, and facial emotion processing in schizophrenia (Fig. 2). ${ }^{76}$ This finding may indicate that CR employing variable training methods elicits neural plasticity in brain areas that underlie cognitive processes. Therefore, it is possible that $\mathrm{CR}$ can improve cognition and functioning by enhancing both neuroplasticity in these brain regions and motivation for recovery.

Nonetheless, considerable efforts are required to develop feasible treatment methods that support recovery of people with schizophrenia, and that particularly target the two persistent and distressing symptoms of negative symptoms and cognitive deficits.

Acknowledgments: The author thanks Drs. Pu and Itakura for critically reading the manuscript.

The author declares no conflict of interest. 


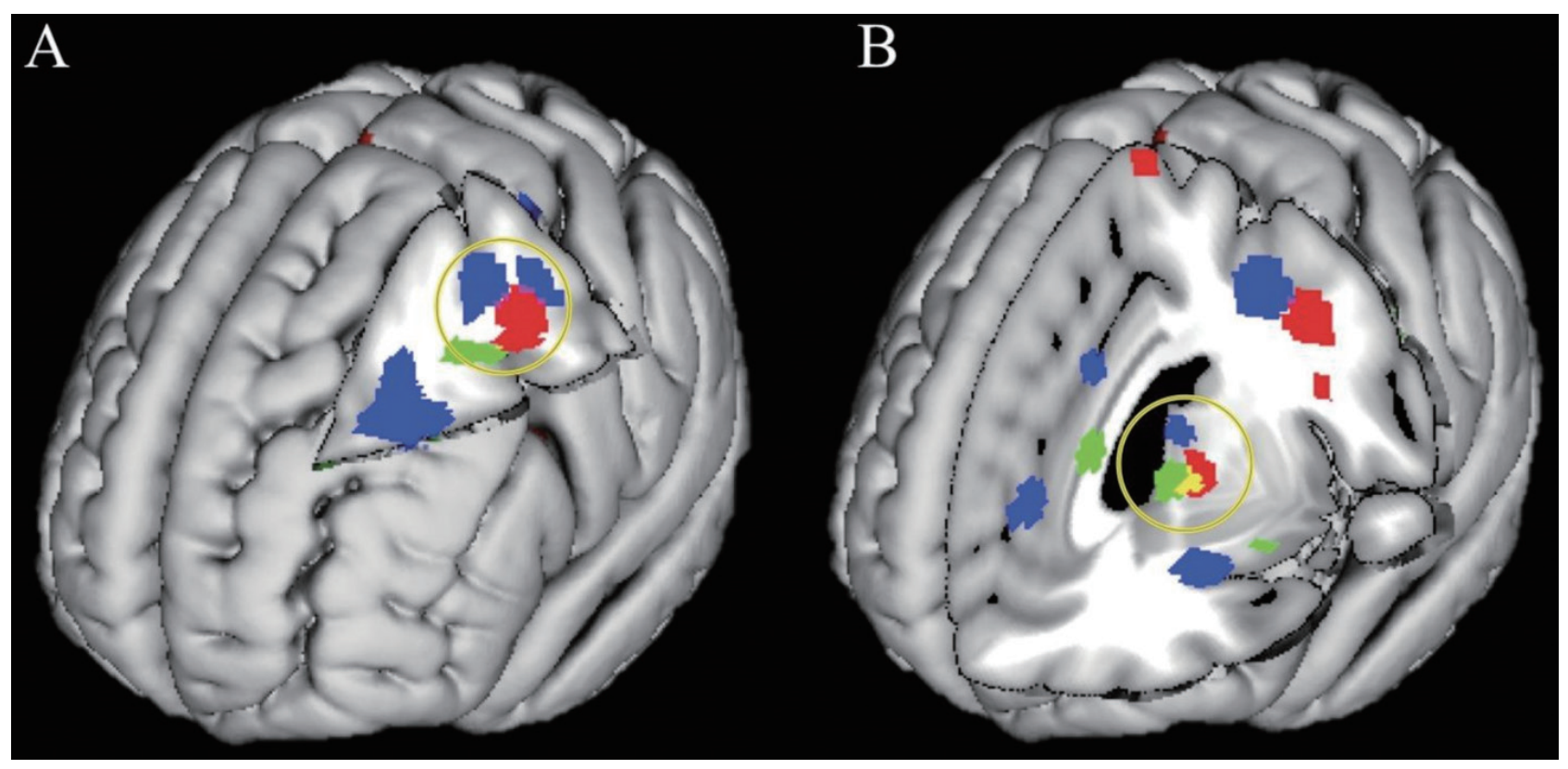

Fig. 2. Cognitive remediation (CR)-elicited changes in brain activation showing that $C R$ training supports restorative functioning in prefrontal and thalamic areas (with permission of Oxford University Press provided February 19, 2018)

(A) Increased CR-elicited functional activation in the left prefrontal cortex overlaps with areas shown to have dysfunctional processing in previous cognition and affective processing activation likelihood estimation (ALE) meta-analyses.

(B) Increased CR-elicited functional activation in the thalamus and caudate nucleus overlaps with an area showing deficits in affective processing and is adjacent to a thalamic area showing deficits in working memory (WM) ALE.

Note: Areas in red depict brain regions that exhibited significant CR-elicited changes in the current ALE. Blue areas depict previously published results comparing healthy controls $(\mathrm{HC})>$ patients with schizophrenia (SZ) on tasks measuring working memory (WM) and executive functioning. Green areas depict previously published results comparing $\mathrm{HC}>\mathrm{SZ}$ on tasks measuring affective processing. Purple indicates overlap between CR and the WM ALE, and yellow indicates overlap between CR and the affective processing ALE.

\section{REFERENCES}

1 Leucht S, Pitschel-Walz G, Abraham D, Kissling W. Efficacy and extrapyramidal side-effects of the new antipsychotics olanzapine, quetiapine, risperidone, and sertindole compared to conventional antipsychotics and placebo. A meta-analysis of randomized controlled trials. Schizophr Res. 1999;35:5168. PMID: 9988841.

2 Keefe RSE, Sweeney JA, Gu H, Hamer RM, Perkins DO, McEvoy JP, et al. Effects of olanzapine, quetiapine, and risperidone on neurocognitive function in early psychosis: a randomized, double-blind 52-week comparison. Am J Psychiatry. 2007a;164:1061-71. PMID: 17606658.

3 Keefe RS, Bilder RM, Davis SM, Harvey PD, Palmer BW, Gold JM, et al. Neurocognitive effects of antipsychotic medications in patients with chronic schizophrenia in the CATIE trial. Arch Gen Psychiatry. 2007b;64:633-47. PMID: 17548746.

4 Green MF, Nuechterlein KH. Should schizophrenia be treated as a neurocognitive disorder? Schizophr Bull. 1999;25:309-19. PMID: 10416733.

5 Green MF, Kern RS, Braff DL, Mintz J. Neurocognitive deficits and functional outcome in schizophrenia: are we measuring the "right stuff"? Schizophr Bull. 2000;26:119-36. PMID: 10755673.

6 Kirkpatrick B, Buchanan RW, Ross DE, Carpenter Jr WT. A separate disease within the syndrome of schizophrenia. Arch
Gen Psychiatry. 2001;58:165-171. PMID: 11177118.

7 Carbon M, Correll CU. Thinking and acting beyond the positive: the role of the cognitive and negative symptoms in schizophrenia. CNS Spectr. 2014;19 Suppl S1:35-53. PMID: 25403863

8 Kirkpatrick B, Fenton WS, Carpenter Jr WT, Marder SR. The NIMH-MATRICS consensus statement on negative symptoms. Schizophr Bull. 2006;32:214-9. PMID: 16481659.

9 Blanchard JJ, Cohen AS. The structure of negative symptoms within schizophrenia: implications for assessment. Schizophr Bull. 2006;32:238-245. PMID: 16254064.

10 Millan MJ, Fone K, Steckler T, Horan WP. Negative symptoms of schizophrenia: clinical characteristics, pathophysiological substrates, experimental models and prospects for improved treatment. Eur Neuropsychopharmacol. 2014;24:64592. PMID: 24820238.

11 Marder SR, Kirkpatrick B. Defining and measuring negative symptoms of schizophrenia in clinical trials. Eur Neuropsychopharmacol. 2014;24:737-43. PMID: 24275698.

12 Andreasen NC. Negative symptoms in schizophrenia. Definition and reliability. Arch Gen Psychiatry. 1982;39:7848. PMID: 7165477.

13 Kay SR, Fiszbein A, Opler LA. The positive and negative syndrome scale (PANSS) for schizophrenia. Schizophr Bull. 1987;13:261-76. PMID: 3616518.

14 Kirkpatrick B, Strauss GP, Nguyen L, Fischer BA, Daniel DG, Cienfuegos A et al. The brief negative symptom scale: psy- 
chometric properties. Schizophr Bull. 2011;37:300-5. PMID: 20558531.

15 Kring AM, Gur RE, Blanchard JJ, Horan WP, Reise SP. The Clinical Assessment Interview for Negative Symptoms (CAINS): final development and validation. Am J Psychiatry. 2013;170:165-72. PMID: 23377637.

16 Strauss GP, Gold JM. A new perspective on anhedonia in schizophrenia. Am J Psychiatry. 2012;169:364-73. PMID: 22407079.

17 Strauss GP, Gold JM. A Psychometric Comparison of the Clinical Assessment Interview for Negative Symptoms and the Brief Negative Symptom Scale. Schizophr Bull. 2016;42:1384-94. PMID: 27174556.

18 Green MF, Hellemann G, Horan WP, Lee J, Wynn JK. From perception to functional outcome in schizophrenia: modeling the role of ability and motivation. Arch Gen Psychiatry. 2012;69:1216-24. PMID: 23026889.

19 Davis MC, Horan WP, Marder SR. Psychopharmacology of the negative symptoms: current status and prospects for progress. Eur Neuropsychopharmacol. 2014;24:788-99. PMID: 24252823.

20 Velthorst E, Koeter M, van der Gaag M, Nieman DH, Fett AK, Smit F, et al. Adapted cognitive-behavioural therapy required for targeting negative symptoms in schizophrenia: meta-analysis and meta-regression. Psychol Med. 2015;45:45365. PMID: 24993642.

21 Fusar-Poli P, Papanastasiou E, Stahl D, Rocchetti M, Carpenter W, Shergill S, et al. Treatments of Negative Symptoms in Schizophrenia: Meta-Analysis of 168 Randomized Placebo-Controlled Trials. Schizophr Bull. 2015;41:892-9. PMID: 25528757.

22 Lieberman JA, Stroup TS, McEvoy JP, Swartz MS, Rosenheck RA, Perkins DO, et al. Effectiveness of antipsychotic drugs in patients with chronic schizophrenia. N Engl J Med. 2005;353:1209-23. PMID: 16172203.

23 Singh SP, Singh V, Kar N, Chan K. Efficacy of antidepressants in treating the negative symptoms of chronic schizophrenia: meta-analysis. Br J Psychiatry. 2010;197:174-9. PMID: 20807960.

24 Rummel C, Kissling W, Leucht S. Antidepressants as addon treatment to antipsychotics for people with schizophrenia and pronounced negative symptoms: a systematic review of randomized trials. Schizophr Res. 2005;80:85-97. PMID: 16183258.

25 Moghaddam B, Javitt D. From revolution to evolution: the glutamate hypothesis of schizophrenia and its implication for treatment. Neuropsychopharmacol. 2012;37:4-15. PMID: 21956446.

26 Rowland LM, Bustillo JR, Mullins PG, Jung RE, Lenroot R, Landgraf E, et al. Effects of ketamine on anterior cingulate glutamate metabolism in healthy humans: a 4-T proton MRS study. Am J Psychiatry. 2005;162:394-6. PMID: 15677610.

27 Krystal JH, Perry EB Jr, Gueorguieva R, Belger A, Madonick $\mathrm{SH}$, Abi-Dargham A, et al. Comparative and interactive human psychopharmacologic effects of ketamine and amphetamine: implications for glutamatergic and dopaminergic model psychoses and cognitive function. Arch Gen Psychiatry. 2005;62:985-94. PMID: 16143730.

28 Singh SP, Singh V. Meta-analysis of the efficacy of adjunctive NMDA receptor modulators in chronic schizophrenia. CNS Drugs. 2011;25:859-85. PMID: 21936588.

29 Goff DC. D-cycloserine: an evolving role in learning and neuroplasticity in schizophrenia. Schizophr Bull. 2012;38:936-41.
PMID: 22368237.

30 Abi-Dargham A, Moore H. Prefrontal DA transmission at D1 receptors and the pathology of schizophrenia. Neuroscientist. 2003;9:404-16. PMID: 14580124.

31 Lindenmayer JP, Nasrallah H, Pucci M, James S, Citrome L. A systematic review of psychostimulant treatment of negative symptoms of schizophrenia: challenging and therapeutic opportunities. Schizophr Res. 2013;147:241-52. PMID: 23619055.

32 Zolkowska D, Jain R, Rothman RB, Prtilla JS, Roth BL, Setola V, et al. Evidence for the involvement of dopamine transporters in behavioral stimulant effects of modafinil. J Pharmacol Exp Ther. 2009;329:738-46. PMID: 19197004.

33 Liberman RP. Rehabilitation as the road to recovery. In: Liberman RP. Recovery from disability. Washington DC: American Psychiatric Publishing; 2008. p. 2-36.

34 Tsapakis EM, Dimopoulou T, Tarazi FI. Clinical management of negative symptoms of schizophrenia: an update. Pharmacol \& Ther. 2015;153:135-47. PMID: 26116809.

35 Oorschot M, Lataster T, Thewissen V, Lardinois M, Wichers $\mathrm{M}$, van Os J, et al. Emotional experience in negative symptoms of schizophrenia-no evidence for a generalized hedonic deficit. Schizophr Bull. 2013;39:217-25. PMID: 22021660.

36 Turkington D, Kingdon D, Weiden PJ. Cognitive behavior therapy for schizophrenia. Am J Psychiatry. 2006;163:363-73. PMID: 16513854.

37 Wykes T, Steel C, Everitt B, Tarrier N. Cognitive behavior therapy for schizophrenia: effect sizes, clinical models, and methodological rigor. Schizophr Bull. 2008;34:523-37. PMID: 17962231.

38 Mueser KT, Deavers F, Penn DL, Cassisi JE. Psychosocial treatments for schizophrenia. Annu Rev Clin Psychol. 2013;9:465-97. PMID: 23330939.

39 Jauhar S, McKenna PJ, Radua J, Fung E, Salvador R, Laws KR. Cognitive-behavioural therapy for the symptoms of schizophrenia: systematic review and meta-analysis with examination of potential bias. Br J Psychiatry. 2014;204:20-9. PMID: 24385461.

40 Grant PM, Huh GA, Perivoliotis D, Stolar NM, Beck AT. Randomized trial to evaluate the efficacy of cognitive therapy for low-functioning patients with schizophrenia. Arch Gen Psychiatry. 2012;69:121-7. PMID: 21969420.

41 Grant PM, Beck AT. Defeatist beliefs as a mediator of cognitive impairment, negative symptoms, and functioning in schizophrenia. Schizophr Bull. 2009;35:798-806. PMID: 18308717.

42 Reichenberg A, Harvey PD. Neuropsychological impairments in schizophrenia: Integration of performance-based and brain imaging findings. Psychol Bull. 2007;133:833-58. PMID: 17723032.

43 Palmer BW, Heaton RK, Paulsen JS, Kuck J, Braff D, Harris $\mathrm{MJ}$, et al. Is it possible to be schizophrenic yet neuropsychologically normal? Neuropsychol. 1997;11:437-46. PMID: 9223148.

44 Green MF. What are the functional consequences of neurocognitive deficits in schizophrenia? Am J Psychiatry. 1996;153:321-30. PMID: 8610818.

45 Hoff AL, Sakuma M, Wieneke M, Horon R, Kushner M, DeLisi LE. Longitudinal neuropsychological follow-up study of patients with first-episode schizophrenia. Am J Psychiatry. 1999;156:1336-41. PMID: 10484942.

46 Bilder RM, Goldman RS, Robinson D, Reiter G, Bell L, Bates JA, et al. Neuropsychology of first-episode schizophrenia: ini- 
tial characterization and clinical correlates. Am J Psychiatry. 2000;157:549-59. PMID: 10739413.

47 Fusar-Poli P, Deste G, Smieskova R, Barlati S, Yung AR, Howes $\mathrm{O}$, et al. Cognitive functioning in prodromal psychosis: a meta-analysis. Arch Gen Psychiatry. 2012;69:562-71. PMID: 22664547.

48 Bora E, Murray RM. Meta-analysis of cognitive deficits in ultra-high risk to psychosis and first-episode psychosis: do the cognitive deficits progress over, or after, the onset of psychosis? Schizophr Bull. 2014;40:744-55. PMID: 23770934.

49 Buchanan RW, Strauss ME, Breier A, Kirkpatrick B, Carpenter WT Jr. Attentional impairments in deficit and nondeficit forms of schizophrenia. Am J Psychiatry. 1997;154:36370. PMID: 9054784.

50 Dominguez Mde G, Viechtbauer W, Simons CJ, van Os J, Krabbendam L. Are psychotic psychopathology and neurocognition orthogonal? A systematic review of their associations. Psychol Bull. 2009;135:157-71. PMID: 19210058.

51 Bratti IM, Bilder RM. Neurocognitive deficits and firstepisode schizophrenia: characterization and course. In: Sharma T and Harvey PD, editors. The early course of schizophrenia. Oxford: Oxford University Press; 2006. p. 87-110.

52 Heinrichs RW, Zakzanis KK. Neurocognitive deficit in schizophrenia: a quantitative review of the evidence. Neuropsychology. 1998;12:426-55. PMID: 9673998.

53 Dickinson D, Ramsey ME, Gold JM. Overlooking the obvious: a meta-analytic comparison of digit symbol coding tasks and other cognitive measures in schizophrenia. Arch Gen Psychiatry. 2007;64:532-42. PMID: 17485605.

54 Green MF, Nuechterlein KH, Gold JM, Barch DM, Cohen J, Essock S, et al. Approaching a consensus cognitive battery for clinical trials in schizophrenia: the NIMH-MATRICS conference to select cognitive domains and test criteria. Biol Psychiatry. 2004;56:301-7. PMID: 15336511.

55 Keefe RSE, Harvey PD. Cognitive Impairment in schizophrenia. 2012. In: Geyer MA and Gross G, editors. Novel Antischizophrenia Treatments, Handbook of Experimental Pharmacology 213. Berlin: Springer-Verlag; 2012. p. 11-37.

56 Posner MI, Petersen SE. The attention system of the human brain. Annu Rev Neurosci. 1990;13:25-42. PMID: 2183676.

57 Baddeley AD, Hitch GJ. Working memory. 1974. In: Bower $\mathrm{G}$, editor. Recent advances in learning and motivation volume VIII. New York: Academic Press; 1974. p. 47-90.

58 Goldman-Rakic PS. Working memory dysfunction in schizophrenia. J Neuropsychiatry Clin Neurosci. 1994;6:348-57. PMID: 7841806.

59 Glahn DC, Ragland JD, Abramoff A, Barrett J, Laird AR, Bearden $\mathrm{CE}$, et al. Beyond hypofrontality: a quantitative meta-analysis of functional neuroimaging studies of working memory in schizophrenia. Hum Brain Mapp. 2005;25:60-9. PMID: 15846819.

60 Brekke J, Kay DD, Lee KS, Green MF. Biosocial pathways to functional outcome in schizophrenia. Schizophr Res. 2005;80:213-25. PMID: 16137859.

61 Nuechterlein KH, Green MF, Kern RS, Baade LE, Barch DM, Cohen JD, et al. The MATRICS Consensus Cognitive Battery, part 1: test selection, reliability, and validity. Am J Psychiatry. 2008;165:203-13. PMID: 18172019.

62 Keefe RS, Goldberg TE, Harvey PD, Gold JM, Poe MP, Coughenour L. The Brief Assessment of Cognition in Schizophrenia: reliability, sensitivity, and comparison with a standard neurocognitive battery. Schizophr Res. 2004;68;283-
97. PMID: 15099610.

63 Takizawa R, Kasai K, Kawakubo Y, Marumo K, Kawasaki $\mathrm{S}$, Yamasue $\mathrm{H}$, et al. Reduced frontopolar activation during verbal fluency task in schizophrenia: a multi-channel nearinfrared spectroscopy study. Schizophr Res. 2008;99:250-62. PMID: 18063344.

64 Pu S, Nakagome K, Itakura M, Yamanashi T, Sugie T, Miura A, et al. Self-reported social functioning and prefrontal hemodynamic responses during a cognitive task in schizophrenia. Psychiatry Res. 2015;234:121-9. PMID: 26382107.

65 Itakura M, Pu S, Ohdachi H, Matsumura H, Yokoyama K, Nagata I, et al. Association between social functioning and prefrontal cortex function during a verbal fluency task in schizophrenia: A near-infrared spectroscopic study. Psychiatry Clin Neurosci. 2017;71:769-79. PMID: 28657683.

66 Davidson M, Galderisi S, Weiser M, Werbeloff N, Fleischhacker WW, Keefe RS, et al. Cognitive effects of antipsychotic drugs in first-episode schizophrenia and schizophreniform disorder: a randomized, open-label clinical trial (EUFEST). Am J Psychiatry. 2009;166:675-82. PMID: 19369319.

67 Medalia A, Saperstein AM. Does cognitive remediation for schizophrenia improve functional outcomes? Curr Opin Psychiatry. 2013;26:151-7. PMID: 23318663.

68 McGurk SR, Twamley EW, Sitzer DI, McHugo GJ, Mueser KT. A meta-analysis of cognitive remediation in schizophrenia. Am J Psychiatry. 2007;164;1791-1802. PMID: 18056233.

69 Wykes T, Huddy V, Cellard C, McGurk SR, Czobor P. A meta-analysis of cognitive remediation for schizophrenia: methodology and effect sizes. Am J Psychiatry. 2011;168:47285. PMID: 21406461.

70 Kurtz MM, Moberg PJ, Gur RC, Gur RE. Approaches to cognitive remediation of neuropsychological deficits in schizophrenia: a review and meta-analysis. Neuropsychol Rev. 2001;11:197-210. PMID: 11883669.

71 Bell M, Bryson G, Wexler BE. Cognitive remediation of working memory deficits: durability of training effects in severely impaired and less severely impaired schizophrenia. Acta Psychiatr Scand. 2003;108:101-9. PMID: 12823166.

72 Thorsen AL, Johansson K, Løberg E-M. Neurobiology of cognitive remediation therapy for schizophrenia: a systematic review. Front Psychiatry. 2014; 5:103. doi: 10.3389/ fpsyt.2014.00103. PMID: 25177300.

$73 \mathrm{Pu}$ S, Nakagome K, Yamada T, Ikezawa S, Itakura M, Satake T, et al. A pilot study on the effects of cognitive remediation on hemodynamic responses in the prefrontal cortices of patients with schizophrenia: a multi-channel near-infrared spectroscopy study. Schizophr Res. 2014;153:87-95. PMID: 24556471.

74 Eack SM, Hogarty GE, Cho RY, Prasad KM, Greenwald DP, Hogarty SS, et al. Neuroprotective effects of cognitive enhancement therapy against gray matter loss in early schizophrenia: results from a 2-year randomized controlled trial. Arch Gen Psychiatry. 2010;67:674-82. PMID: 20439824.

75 Penadés R, Pujol N, Catalán R, Massana G, Rametti G, García-Rizo C, et al. Brain effects of cognitive remediation therapy in schizophrenia: a structural and functional neuroimaging study. Biol Psychiatry. 2013;73:1015-23. PMID: 23452665 .

76 Ramsay IS, MacDonald AW III. Brain correlates of cognitive remediation in schizophrenia: Activation Likelihood Analysis shows preliminary evidence of neural target engagement. Schizophr Bull. 2015;41:1276-84. PMID: 25800249. 